\title{
Influence of Buffers and Culture Media on Diamond Solution-Gated Field Effect Transistors Regarding Stability and Memory Effect ${ }^{\dagger}$
}

\author{
Václav Procházka ${ }^{1,2, *}$, Tibor Ižák ${ }^{1}$ and Alexander Kromka ${ }^{1,3}$ \\ 1 Institute of Physics, Czech Academy of Sciences v.v.i., 16200 Prague, Czech Republic; izak@fzu.cz (T.I.); \\ kromka@fzu.cz (A.K.) \\ 2 Faculty of Electrical Engineering, Czech Technical University in Prague, 16627 Prague, Czech Republic \\ 3 Faculty of Civil Engineering, Czech Technical University in Prague, 16629 Prague, Czech Republic \\ * Correspondence: prochazkav@fzu.cz; Tel.: +420-220-318-511 \\ + Presented at the Eurosensors 2017 Conference, Paris, France, 3-6 September 2017.
}

Published: 25 August 2017

\begin{abstract}
The transfer characteristics of a nanocrystalline diamond (NCD)-based solution-gated field effect transistor (SGFET) under the influence of inorganic and organic compounds were studied. Studied compounds included three different buffer solutions (Phosphate, HEPES, Mcllvaine buffer) and commonly used culture media (fibronectin, albumin and fetal bovine serum). It was found that buffers with the same $\mathrm{pH}$ of 7.4 caused different voltage shifts in transfer characteristics. This effect was reversible which indicates the surface stability of the hydrogen-terminated diamond during repeated measurements. In contrast to this observation, the SGFET sensitivity decreased after applying the culture solutions which we attribute to the permanently adsorbed bio-layer formed on the SGFET channel sensing area.
\end{abstract}

Keywords: nanocrystalline diamond; field-effect transistor; transfer characteristics

\section{Introduction}

Development of cell sensitive biosensors is a relatively young science discipline. Its evolution in recent years is closely tied to the development of technology, especially the integration and miniaturisation of electronic components. Sensors of cell cultures and tissues place considerable emphasis on chemical inertness, surface morphology and non-invasiveness of the sensing material, all of which are included in the term of biocompatibility. Apart from a large family of such materials, diamond is recognized as an outstanding material regarding long-term biocompatibility.

In past few years, a new generation of carbon-based bio-electronic devices based on p-type surface conductive thin films was developed. For example, solution-gated field effect transistors (SGFETs) based on carbon nanotubes for direct detection of Heroin metabolites were developed [1]; or graphene coatings were widely studied for $\mathrm{pH}$ [2], DNA or glucose sensing applications [3]. Last but not least carbon material, a diamond film opened new perspectives in the field of tissue engineering, prosthetics and microbiology. Hydrogen-terminated diamond exhibits induced surface conductivity, which is highly sensitive to changes in the surrounding environment due to surface transfer doping mechanism [4]. Multiple studies of diamond SGFETs have carried out regarding their $\mathrm{pH}$ or ion sensitivity [5] and ongoing effect at the protein-diamond interface [6]. Amongst other electrical properties, gate currents and cell interface properties were discussed [7]. In the present study, we discuss the influence of several buffers and culture solutions on electrical characteristics of the diamond SGFETs. Such measurements are essential for the complex studies of mammalian cell culture apoptosis, whereas unclear sensor responses may occur during the cell necrosis. 


\section{Materials and Methods}

The active sensor area of SGFETs was realised by hydrogen-terminated nanocrystalline diamond (NCD) thin film which exhibits subsurface p-type conductive channel. NCD films were grown in a microwave ellipsoidal cavity reactor by chemical vapor deposition process for $4.5 \mathrm{~h}$, at following process conditions: pressure $30 \mathrm{mbar}$, gas mixture $1 \% \mathrm{CH}_{4}$ in $\mathrm{H}_{2}$, microwave power $1500 \mathrm{~W}$, deposition temperature $550{ }^{\circ} \mathrm{C}$. Diamond films were further processed for $10 \mathrm{~min}$ in the same reactor in hydrogen plasma to induce p-type surface conductivity.

Electrical measurements were performed using $\mathrm{Ag} / \mathrm{AgCl}$ gate electrode setup described in [8]. The SGFET sensing area was flooded by buffer or culture solution ( $15 \mu \mathrm{L}$ droplet) in a specific sequence (see Figure $1 \mathrm{~b} x$-axes "Measurement order") and gated with $\mathrm{Ag} / \mathrm{AgCl}$ gate electrode. Three different buffers (Phosphate (PBS), HEPES and Mcllvaine buffer) with the same $\mathrm{pH}$ value of 7.4 and three culture media (fibronectin (FN), albumin (BSA) and fetal bovine serum (FBS)) were measured. Before each $\mathrm{I}-\mathrm{V}$ measurement, the sensing area was rinsed with deionized water (DIW) and dried under nitrogen flow. The layout of the diamond-based SGFET sensor is similar to sensors used in [7].

a)

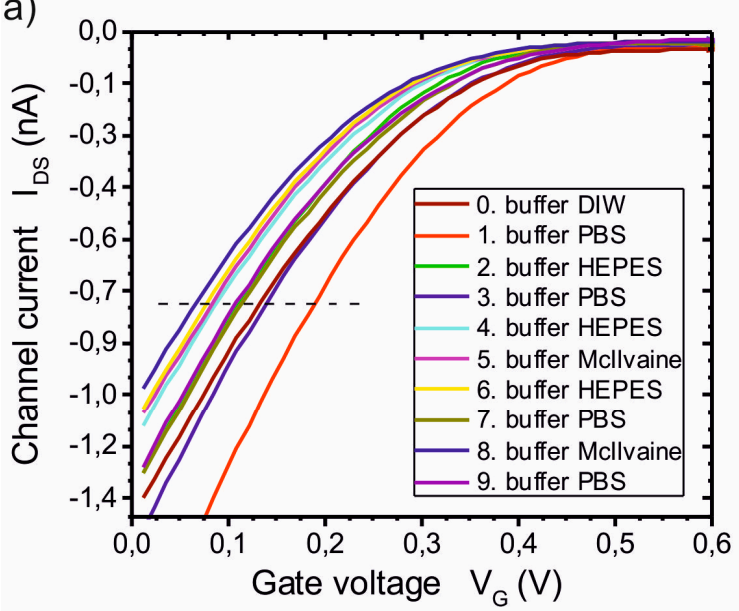

b)

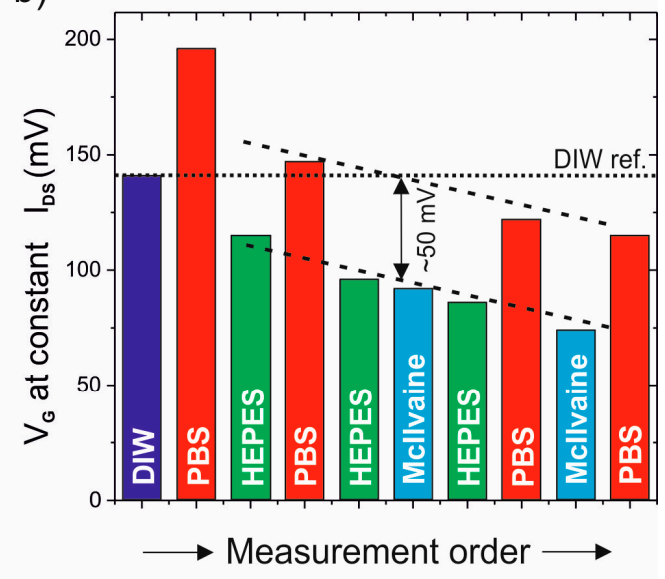

Figure 1. (a) Transfer characteristics of SGFET measured with tree buffers; (b) Gate voltage shifts as a function of various buffer solutions measured constant IDs current at $0.75 \mathrm{nA}$.

\section{Results and Discussion}

The transfer characteristics of diamond SGFET as a function of different buffer solutions and measurements order are shown in Figure 1a. For better illustration and interpretation of dependencies, the gate voltages at constant current (in our case IDs $=0.75 \mathrm{nA}$, see the dotted line in Figure 1a are plotted in columnar graphs (Figure 1b)). It should be noted that the gate voltage shifts refer to the voltage measured for deionized water (DIW ref).

As it is shown in Figure 1b, PBS buffer caused a positive gate voltage shift of $+59 \mathrm{mV}$ referred to DIW. On the other hand, HEPES and McIlvaine buffers caused the voltage shifts of $-52 \mathrm{mV}$ and $-49 \mathrm{mV}$ (referred to PBS), respectively. The modulation of SGFET characteristics with buffers can be caused either by so-called electric field effect [9] or by the change of counter ions concentration at the channel surface [10]. In our case, the origin of gate voltage shifts we attribute to the variation of the counter ions at the channel surface, i.e., to the decrease of counter ions concentration, thus to the closing of the p-type channel. We attributed this effect to the presence of disodium phosphate ions. Compared to PBS, HEPES and McIlvaine buffers contain larger molecules, which possibly increase the counter ions concentration in the close vicinity of the channel, resulting in the negative shifts.

Another advantage of the presented diamond-based SGFET is its stability. Repetitive use of SGFETs, its rinsing in DIW and exposing to a specific buffer, did not degrade its functionality. As shown in Figure $1 b$, the voltage shift $\left(\Delta V_{G}\right)$ between PBS and HEPES (or Mcllvaine) buffer is nearly constant; only the $\Delta V_{\mathrm{G}}$ referred to DIW decreases (see decreasing two parallel dotted lines). This 
dependence indicates that the H-terminated diamond surface is stable enough, i.e., does not chemically degrade by oxidation, etc. It should be noted that similar transfer characteristics were also measured for different culture media (not shown here). In this case, positive voltage shifts of $+17 \mathrm{mV}$ and $+13 \mathrm{mV}$ were obtained for BSA and FN. However, after repeated applications of BSA, the shift decreased to $+8 \mathrm{mV}$, while for FN the voltage shifts further increased to $+18 \mathrm{mV}$. Although, the clear tendency was not observed. It is well known that FN adheres on a hydrophobic surface and deposits a non-washable bio-layer [6]. We assume that such bio-layer formed on the surface will reveal an additional increase of the gate currents. Our measurements confirmed a memory effect of the SGFET [11]. Moreover, FBS irreversibly changed the diamond/solution interface. Exposing diamond SGFETs to culture solutions seems to be a more complex issue.

\section{Conclusions}

Preliminary results confirmed that optically transparent and biocompatible diamond-based SGFET is a promising platform for real-time monitoring of cell cultures. Varied and repeated measurements with different buffer solutions showed high reversibility. The gate voltage shift between different buffers (in this case PBS and HEPES or Mcllvaine) was constant ( $50 \mathrm{mV})$, only the relative value of the voltage (referred to the voltage measured at first application of sensor) tended to decrease. This effect is related to the surface stability of the hydrogen-terminated diamond during repeated measurements. In the case of culture media, the sensor response was most probably disrupted by the adsorbed bio-layer. Further experiments are in progress to deeper understand the SGFETs reproducibility and repeatability on culture media.

Acknowledgments: This work was supported by Grant Agency of Czech Republic grant No. P108/12/G108 and Czech Technical University grant No. SGS17/136/OHK4/2T/13 (VP).

Conflicts of Interest: The authors declare no conflict of interest.

\section{References}

1. Mhaisalkar, S.G.; Tey, J.N.; Gandhi, S.; Wijaya, I.P.M.; Palaniappan, A.; Wei, J.; Rodriguez, I.; Suri, C.R. Direct detection of heroin metabolites using a competitive immunoassay based on a carbon-nanotube liquid-gated field-effect transistor. Small 2010, 6, 993-998, doi:10.1002/smll.200902139.

2. Ang, P.K.; Chen, W.; Wee, A.T.S.; Kian, P.L. Solution-gated epitaxial graphene as $\mathrm{pH}$ sensor. J. Am. Chem. Soc. 2008, 130, 14392-14393, doi:10.1021/ja805090z.

3. Zhang, M.; Liao, C.; Mak, C.H.; You, P.; Mak, C.L.; Yan, F. Highly sensitive glucose sensors based on enzyme-modified whole-graphene solution-gated transistors. Sci. Rep. 2015, 5, 8311, doi:10.1038/srep08311.

4. Strobel, P.; Riedel, M.; Ristein, J.; Ley, L.; Boltalina, O. Surface transfer doping of diamond by fullerene. Diam. Relat. Mater. 2005, 14, 451-458, doi:10.1016/j.diamond.2004.12.051.

5. Lee, C.S.; Kyu Kim, S.; Kim, M. Ion-sensitive field-effect transistor for biological sensing. Sensors 2009, 9, 7111-7131, doi:10.3390/s90907111.

6. Roach, P.; Farrar, D.; Perry, C.C. Interpretation of protein adsorption: Surface-induced conformational changes. J. Am. Chem. Soc. 2005, 127, 8168-8173, doi:10.1021/ja0428980.

7. Ižák, T.; Krátká, M.; Kromka, A.; Rezek, B. Osteoblastic cells trigger gate currents on nanocrystalline diamond transistor. Colloids Surf. B Biointerfaces 2015, 129, 95-99, doi:10.1016/j.colsurfb.2015.03.035.

8. Procházka, V.; Cifra, M.; Kulha, P.; Ižák, T.; Rezek, B.; Kromka, A. Influence of non-adherent yeast cells on electrical characteristics of diamond-based field-effect transistors. Appl. Surf. Sci. 2017, 395, 214-219, doi:10.1016/j.apsusc.2016.05.003.

9. Wang, X.; Kurihara, S.; Hasegawa, M.; Ruslinda, A.R.; Kawarada, H. High priority of nanocrystalline diamond as a biosensing platform. Jpn. J. Appl. Phys. 2012, 51, 090125, doi:10.1143/JJAP.51.090125. 
10. Krátká, M.; Babchenko, O.; Ukraintsev, E.; Vachelová, J.; Davídková, M.; Vandrovcová, M.; Kromka, A.; Rezek, B. Gamma radiation effects on hydrogen-terminated nanocrystalline diamond bio-transistors. Diam. Relat. Mater. 2016, 63, 186-191, doi:10.1016/j.diamond.2015.10.015.

11. Liskova, J.; Babchenko, O.; Varga, M.; Kromka, A.; Hadraba, D.; Svindrych, Z.; Burdikova, Z.; Bacakova, L. Osteogenic cell differentiation on H-terminated and O-terminated nanocrystalline diamond films. Int. J. Nanomed. 2015, 10, 869-884, doi:10.2147/IJN.S73628.

(C) 2017 by the authors. Licensee MDPI, Basel, Switzerland. This article is an open access article distributed under the terms and conditions of the Creative Commons Attribution (CC BY) license (http://creativecommons.org/licenses/by/4.0/). 